\title{
Negative modes of Coleman-De Luccia and black hole bubbles
}

\author{
Ruth Gregory, ${ }^{1,2, *}$ Katie M. Marshall, ${ }^{3, \dagger}$ Florent Michel, ${ }^{1, \hbar}$ and Ian G. Moss ${ }^{3, \S}$ \\ ${ }^{1}$ Centre for Particle Theory, Durham University, South Road, Durham DH1 3LE, United Kingdom \\ ${ }^{2}$ Perimeter Institute, 31 Caroline Street North, Waterloo, Ontario N2L 2Y5, Canada \\ ${ }^{3}$ School of Mathematics, Statistics and Physics, Newcastle University, \\ Newcastle Upon Tyne NE1 7RU, United Kingdom
}

(Received 7 August 2018; published 19 October 2018)

\begin{abstract}
We study the negative modes of gravitational instantons representing vacuum decay in asymptotically flat space-time. We consider two different vacuum decay scenarios: the Coleman-de Luccia O(4)symmetric bubble, and $\mathrm{O}(3) \times \mathbb{R}$ instantons with a static black hole. In spite of the similarities between the models, we find qualitatively different behaviors. In the $\mathrm{O}(4)$-symmetric case, the number of negative modes is known to be either one or infinite, depending on the sign of the kinetic term in the quadratic action. In contrast, solving the mode equation numerically for the static black hole instanton, we find only one negative mode with the kinetic term always positive outside the event horizon. The absence of additional negative modes supports the interpretation of these solutions as giving the tunneling rate for false vacuum decay seeded by microscopic black holes.
\end{abstract}

DOI: 10.1103/PhysRevD.98.085017

\section{INTRODUCTION}

False vacuum decay through the nucleation of true vacuum bubbles has many important applications ranging from early universe phase transitions to stability of the Higgs vacuum. The process has an elegant description in terms of Euclidean solutions to the underlying field equations that extend the bubbles into imaginary time [1,2]. Path integral methods give vacuum decay rates which depend on the Euclidean action of the bubble solutions and the eigenvalues of perturbative modes on the bubble background.

A crucial feature of the bubble nucleation picture is the existence of a single negative mode of field perturbations, which corresponds physically to scaling the size of the bubble up or down. In the analysis of the vacuum decay process, the square root of this negative mode provides an imaginary part to the energy of the false vacuum state, which in turn corresponds to a decay rate. The analysis would fail if, e.g., we have two negative modes in which case the net contribution to the energy becomes real. Fortunately, it is known that the basic picture with a single bubble has just the single negative mode [3].

\footnotetext{
r.a.w.gregory@durham.ac.uk

†k.marshall6@newcastle.ac.uk

florent.c.michel@durham.ac.uk

§ian.moss@newcastle.ac.uk
}

Published by the American Physical Society under the terms of the Creative Commons Attribution 4.0 International license. Further distribution of this work must maintain attribution to the author(s) and the published article's title, journal citation, and DOI. Funded by SCOAP .
Coleman and de Luccia [4] were the first people to extend the basic formalism of vacuum decay to include the effects of gravitational back-reaction in the bubble solutions, producing a type of gravitational instanton. The negative modes of the Coleman-de Luccia instanton that represents vacuum decay in de Sitter space have been studied by several authors [5-15], and there is now compelling evidence that the bounce solutions can have either one or infinitely many negative modes, depending on the potential. The existence of many negative modes seems to be associated with situations where the bounce solution is comparable in size to the cosmological horizon [14].

The past few years have seen a resurgence of interest in applications of vacuum decay to the standard model Higgs field [16-22]. Depending on the values of the Higgs and top quark masses, the quantum corrected Higgs potential can decrease at large field values and destabilise the present day $246 \mathrm{GeV}$ minimum. The scale at which an instability sets in, $\Lambda$, is very sensitive to particle physics parameters and possible new physics, with typical ranges $10^{10}-10^{18} \mathrm{GeV}$ leading to a metastable false vacuum [23-25]. Vacuum decay rates are strongly exponentially suppressed, but recently the possibility of black holes seeding vacuum decay has been considered [26-33] and the decay in this case is very rapid. Its implications for early cosmology have been investigated in [34]. In parallel, it was shown in [35] that Hawking radiation can be described by a family of instantons.

Similar ideas have been discussed in the context of eternal inflation, see for instance [36], as well as [37,38] in which the limit of vanishing cosmological constant in the 
false vacuum phase is studied in details. It is found, in particular, that this limit is continuous, contrary to what was previously conjectured.

The negative mode problem has so far only been investigated numerically for vacuum decay in asymptotically de Sitter spacetimes. In this paper we give the first analysis of negative modes for the asymptotically flat bounces that are relevant for decay of the Higgs vacuum. We look at two different Higgs vacuum decay scenarios, vacuum decay in empty space and vacuum decay seeded by black holes. Vacuum decay rates with gravitational backreaction in empty space have been examined by [20,39-41]. The gravitational back reaction is significant when $\Lambda$ approaches the Planck scale, as might be expected. Nonminimal coupling of the Higgs field to gravity can have a significant effect on the decay process, and so we include this possibility on our negative mode analysis.

For decay in empty space, we find numerically that there is either a single negative mode, or infinitely many as in the de Sitter case. The emergence of the infinite tower of negative modes is related to a change in sign for the kinetic terms in the action of the perturbations. This is also seen in the asymptotically de Sitter case. We have used an approach where the gravitational constraints are used to eliminate extraneous gauge degrees of freedom. Our approach is therefore free of gauge artefacts, and gives similar equations to those in Ref. [14], where a gauge invariant parametrization was used.

The second scenario we have investigated is the case where vacuum decay is enhanced by the presence of a microscopic black hole left over from the early universe. The black hole acts as a nucleation seed and greatly enhances the vacuum decay rate. This effect was investigated initially for vacuum decay in de Sitter space [26], and later for more general scenarios including asymptotically flat space [27-29]. In all cases, the dominant decay process is one with static $\mathrm{O}(3)$ symmetric bubbles. We shall give the results of a numerical analysis of the negative modes for vacuum decay with an asymptotically flat black hole nucleation seed. In this case we find only one negative mode, and the kinetic term in the action of the perturbations is always positive. We conclude from this that vacuum decay seeded by black holes most likely always has a consistent formulation in terms of bounce solutions.

\section{TUNNELING AND NEGATIVE MODES}

We consider decay of the false vacuum state of a scalar field $\phi$ with potential $V(\phi)$. Tunneling from the false vacuum is represented in the path integral formalism by bounce solutions $\phi_{b}$ to the scalar field equations, with imaginary time coordinate $\tau$ [1]. Boundary conditions are $\phi_{b} \rightarrow \phi_{\mathrm{fv}}$ when $\tau \rightarrow \pm \infty$ and at spatial infinity $|\mathbf{x}| \rightarrow \infty$, where $\phi_{\mathrm{fv}}$ is the value of the field at the false vacuum. The tunneling exponent for a bounce solution is related to the change in Euclidean action by $B=S_{E}\left[\phi_{b}\right]-S_{E}\left[\phi_{\mathrm{fv}}\right]$, where
$B=\int_{-\infty}^{\infty} d \tau \int d^{3} x\left(\frac{1}{2}\left(\partial_{\tau} \phi_{b}\right)^{2}+\frac{1}{2}\left(\nabla \phi_{b}\right)^{2}+V\left(\phi_{b}\right)\right)$.

Given reasonable conditions on the potential, it has been shown [42] that there is a bubble solution with $O(4)$ symmetry that has the smallest action, and hence the largest tunneling rate, compared to other bounce solutions. Furthermore, this solution has exactly one negative mode [2], and is therefore a saddle point of the Euclidean action.

Evaluating the path integral for a single bubble solution gives a contribution to the vacuum decay amplitude of the form

$$
I_{\text {bubble }} \approx \frac{1}{2} i \Omega T\left|\frac{\operatorname{det}^{\prime} S_{E}^{\prime \prime}\left[\phi_{b}\right]}{\operatorname{det} S_{E}^{\prime \prime}\left[\phi_{\mathrm{fv}}\right]}\right|^{-1 / 2} \frac{B^{2}}{4 \pi^{2}} e^{-B} I_{\mathrm{fv}},
$$

where $S_{E}^{\prime \prime}$ denotes the second functional derivative of the Euclidean action, and $\operatorname{det}^{\prime}$ denotes omission of zero modes from the determinant. The zero modes give factors $\Omega$ and $T$ for the total volume and time period, along with a Jacobian factor $B^{2} / 4 \pi^{2}$. The factor $i$ arises from the negative mode. This would become $i^{n}$ if there were $n$ negative modes. The vacuum decay rate $\Gamma$ can be calculated by summing multiple bubble amplitudes, and the result is $[2,42]$

$$
\Gamma \approx\left|\frac{\operatorname{det}^{\prime} S_{E}^{\prime \prime}\left[\phi_{b}\right]}{\operatorname{det} S_{E}^{\prime \prime}\left[\phi_{\mathrm{fv}}\right]}\right|^{-1 / 2} \frac{B^{2}}{4 \pi^{2}} e^{-B} .
$$

The negative mode can be explained easily in the thinwall limit, when the bubble solution consists of a true vacuum region $\phi_{\mathrm{tv}}$ surrounded by a relatively narrow wall where the field transitions to the false vacuum. This approximation is valid when the difference in energy $\varepsilon$ of the true and false vacua is small compared to a combination of barrier height and width. The field is represented by a bubble Ansatz of the form $\phi=$ $\phi(r ; R) \simeq \phi_{0}(r-R)$, where $\phi_{0}(x)$ solves the "planar" domain wall equation

$$
\phi^{\prime \prime} \approx \frac{\partial V}{\partial \phi} .
$$

Provided the bubble is large compared to the wall thickness this is an excellent approximation, and allows us to integrate the tunneling exponent in terms of the bubble radius $R$,

$$
B(R)=2 \pi^{2} \sigma R^{3}-\frac{1}{2} \pi^{2} \varepsilon R^{4} .
$$

Here, $\sigma$ is the action per unit area of the bubble wall, which can be found in terms of an integral of the potential $V(\phi)$ by

$$
\sigma=\int_{\phi_{\mathrm{fv}}}^{\phi_{\mathrm{tv}}}|2 \Delta V(\phi)|^{1 / 2} d \phi
$$


using $\frac{1}{2} \phi_{0}^{\prime 2}=\Delta V$ from (4). The bubble solution is given by the extremum at the radius $R_{b}=R_{0} \equiv 3 \sigma / \varepsilon$, where $B$ has a maximum.

The negative mode corresponds to changes in $\phi$ that increase or decrease the radius of the bubble solution,

$$
\delta \phi=\frac{d \phi}{d R} \delta R .
$$

The overall change in $B$ is related to the negative eigenvalue $\lambda_{0}$ by,

$$
\delta B \approx \frac{1}{2} B^{\prime \prime}(R) \delta R^{2} \approx \frac{1}{2}\|\delta \phi\|^{2} \lambda_{0},
$$

where the norm of a function $f(x)$ is defined by

$$
\|f\|^{2}=\int f(x)^{2} d^{4} x .
$$

We therefore have a simple formula for the negative mode in the thin-wall approximation,

$$
\left.\lambda_{0} \approx \frac{B(R)^{\prime \prime}}{\|d \phi / d R\|^{2}}\right|_{R=R_{b}},
$$

This can be taken further using our approximation for the bubble wall profile, since $d \phi_{0} / d R=-\phi_{0}{ }^{\prime}$, hence $\|d \phi / d R\|^{2}=\left\|\phi^{\prime}\right\|^{2} \approx 2 \pi^{2} \sigma R^{3}$, and we have

$$
\lambda_{0} \approx-\frac{3}{R_{b}^{2}} \text {. }
$$

The approximation is valid when the thickness of the wall is small compared to the bubble radius, which translates to $\varepsilon \ll 9 \sigma^{2} / \phi_{\mathrm{tv}}^{2}$.

Now we turn to bubble solutions with gravitational backreaction. These can be found by extremizing the Einstein-scalar action,

$$
S_{E}=\int\left(-\frac{\mathcal{R}}{16 \pi G}+\frac{1}{2}(\partial \phi)^{2}+V(\phi)\right) \sqrt{g} d^{4} x,
$$

where $\mathcal{R}$ is the Ricci scalar. Bubble solutions with $O(4)$ symmetry can be described by a "radial" solution of scalar field, $\phi(r)$, and geometry:

$$
d s^{2}=d r^{2}+a^{2}(r) d \Omega_{I I I}^{2}
$$

where $\phi$ and $a$ tend towards the true vacuum form as $r$, $a(r) \rightarrow 0$, and the false vacuum form for large $r$. We take a leap of faith in assuming that the vacuum decay exponent for a single bubble is still given by the difference in Euclidean action between the bubble solution and the false vacuum. There are two distinct scenarios, depending on whether the Euclidean metric is compact or infinite. In the compact case, the scalar field never quite reaches the false vacuum value outside the bubble, but regularity conditions on the metric at the two points where $a=0$ restrict the possible bubble solutions. In the infinite case, the scalar field asymptotically approaches the false vacuum value as $a \rightarrow \infty$. In this case we require that the Euclidean metric approaches the same form for the bounce and for false vacuum to ensure that the tunneling exponent $B=$ $S_{E}\left[\phi_{b}\right]-S_{E}\left[\phi_{\mathrm{fv}}\right]$ is finite. (Note that adding boundary terms to the Einstein-scalar action is unnecessary as these cancel out when evaluating $B$.)

Following Coleman and de Luccia, we gain some insight into the $O(4)$ bubble solutions by taking a thin-wall limit. The thin wall approximation assumes that the scalar varies rapidly between its false and true vacuum over a region $w \ll R$, where $R$ as before represents the size of the bubble radius that we take as the areal radius: the value of the scale factor at the centre of the bubble wall. The thin wall approximation is valid provided that the local spacetime curvature induced within the wall remains below the Planck scale $w \sigma \ll M_{p}^{2}$ [43]. [In this work we use the reduced Planck mass, defined by $M_{p}^{2}=1 /(8 \pi G)$.]

We shall see in the following section that the curvedspace bubble solutions can be represented by the form $\phi=$ $\phi(a ; R)$ where $\phi \approx \phi_{0}\left(r-r_{b}\right)$ for the thin wall, with $r_{b}$ the coordinate location of the bubble center: $a\left(r_{b}\right)=R$. We then approximate the scale factor by a piecewise differentiable function

$$
a(r)=a_{\mathrm{tv}}(r) \Theta\left[r_{b}-r\right]+a_{\mathrm{fv}}(r) \Theta\left[r-r_{b}\right],
$$

where $a_{\mathrm{tv}}\left(r_{b}\right)=a_{\mathrm{fv}}\left(r_{b}\right)=R$, and compute the difference in action between the bubble and false vacuum configurations for this Ansatz. The Ansatz also allows us to estimate the negative eigenvalue as before, but with the norm calculated using an appropriate curved space measure.

Considering first the compact case, we take the false vacuum to have positive energy $\varepsilon$, and the true vacuum to have zero energy. This represents the decay of the false vacuum from a de Sitter universe into flat space, thus $a_{\mathrm{tv}}=r$ and $a_{\mathrm{fv}}=\ell \sin \left(\left(r-r_{0}\right) / \ell\right)$ in (14), where $\ell=$ $\sqrt{3 /(8 \pi G \varepsilon)}$ is the de Sitter radius and $r_{0}$ is a introduced to satisfy $a_{\mathrm{tv}}\left(r_{b}\right)=a_{\mathrm{fv}}\left(r_{b}\right)=R$. The tunneling exponent can be directly calculated as (see also [4])

$$
\begin{aligned}
B(R)= & \frac{4}{3} \pi^{2} \varepsilon \ell^{4}\left\{1 \mp\left(1-R^{2} / \ell^{2}\right)^{3 / 2}\right\} \\
& -2 \pi^{2} \varepsilon \ell^{2} R^{2}+2 \pi^{2} \sigma R^{3},
\end{aligned}
$$

and is plotted in Fig. 1. The upper sign applies when the false vacuum region is larger than a hemisphere, and the lower sign applies when the false vacuum covers less than a hemisphere. In the first case, the true vacuum bubble 

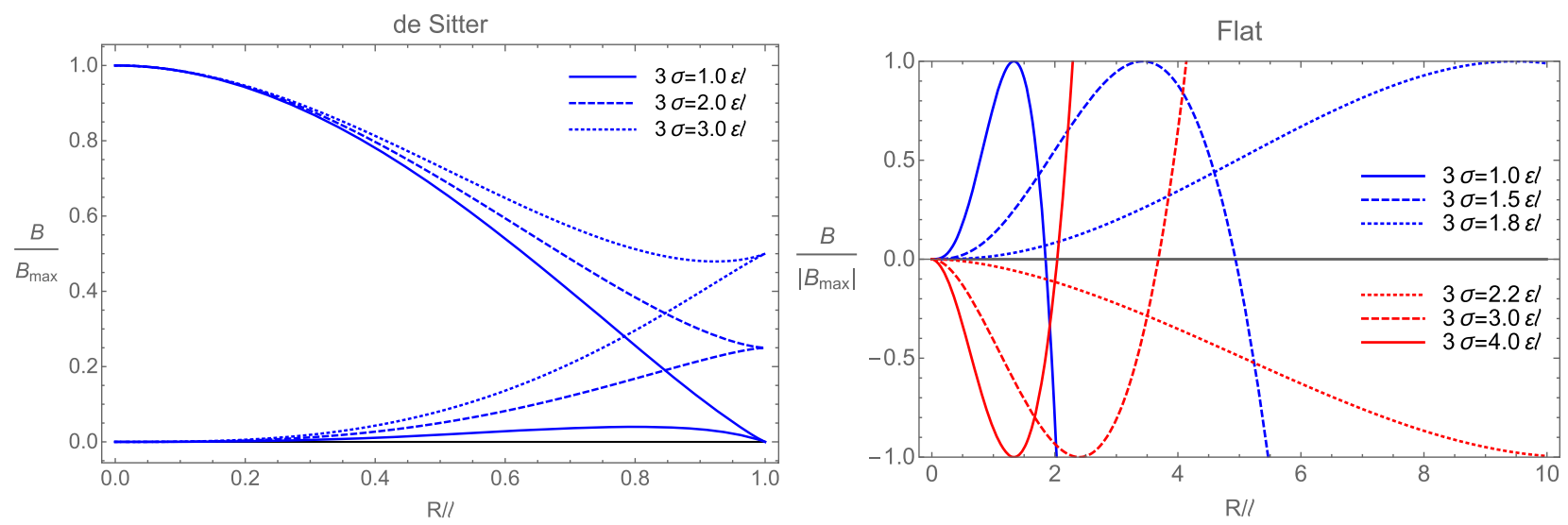

FIG. 1. Left panel: The tunneling exponent $B(R)$ for a thin-wall bubble of flat vacuum in de Sitter space. The large and small bubble exponents are superposed. Right panel: The tunneling exponent $B(R)$ for a thin-wall bubble of anti-de Sitter vacuum in flat space.

encloses a smaller volume than the false vacuum region, and in the second case the true and false vacuum regions have a similar volume. Following Ref. [14], we refer to these as the small bubble and large bubble situations.

The exponent (15) has one extremum $R_{b}$ away from the origin,

$$
R_{b}=\frac{R_{0}}{1+\left(R_{0} / 2 \ell\right)^{2}}
$$

where $R_{0}=3 \sigma / \varepsilon$ is the bubble radius without the gravitational back reaction. Bubble solutions always exist, but the extremum becomes a minimum when $3 \sigma / \varepsilon>2 \ell$. The thin-wall approximation therefore predicts the disappearance of the negative mode, and we can estimate the value of the mode in a similar way to the probe case. Since the bubble wall is determined by $r=R$, and the geometry inside the bubble is flat, we find that the eigenvalue is well approximated here by the flat space value (10). Numerical investigations have shown that new sets of spherically symmetric negative modes start to appear $[5,6,9,14,15]$. The first set are fluctuations localized near the bubble wall, called "wall modes" in Ref. [14]. The second set are localized near the maximum radius of the instanton in the "large bubble" case.

In the noncompact case, the true vacuum has negative energy $-\varepsilon$ and the false vacuum has zero energy. This represents vacuum decay from flat space to anti de Sitter space, and we have $a_{\mathrm{tv}}=\ell \sinh r / \ell, a_{\mathrm{fv}}=r+\left(R-r_{b}\right)$ in (14). This time one finds

$$
\begin{aligned}
B(R)= & \frac{4}{3} \pi^{2} \varepsilon \ell^{4}\left\{1-\left(1+R^{2} / \ell^{2}\right)^{3 / 2}\right\} \\
& \pm 2 \pi^{2} \varepsilon \ell^{2} R^{2}+2 \pi^{2} \sigma R^{3}
\end{aligned}
$$

plotted in the right panel of Fig. 1. The upper sign corresponds to $3 \sigma / \varepsilon<2 \ell$, and the bubble matches an interior of $\mathrm{AdS}$ to an exterior of an $S^{3}$ in $\mathbb{R}^{4}$, i.e., a conventional bubble. For $3 \sigma / \varepsilon>2 \ell$ we can still find a solution, provided we match the interior of an AdS sphere to an interior of a three-sphere in $\mathbb{R}^{4}$ : clearly this does not have an intuitive interpretation as a vacuum decay bubble, and is similar to the situation of dS tunneling above, where the false vacuum covers less than a hemisphere of dS. We note simply that these solutions do not have a negative mode, hence are not tunneling instantons, and do not consider them further. For $3 \sigma / \varepsilon<2 \ell$ the bubble has radius [4]

$$
R_{b}=\frac{R_{0}}{1-\left(R_{0} / 2 \ell\right)^{2}} .
$$

Whenever a bubble solution exists the extremum is always a maximum and the negative mode we had originally should remain. This time, in our estimate of the negative eigenvalue, we note $R=\ell \sinh r_{b} / \ell$, hence

$$
\left\|\frac{d \phi}{d R}\right\|^{2}=\left\|\frac{d \phi}{d r_{b}}\right\|^{2}\left(\frac{d r_{b}}{d R}\right)^{2}=2 \pi^{2} \sigma R^{3}\left(1+R^{2} / \ell^{2}\right)^{-1}
$$

We can substitute this into the general formula (10), with the exponent $B(R)$ from (17), and evaluate the result at the bubble radius $R_{b}$ from (18), to get

$$
\lambda_{0} \approx-\frac{3}{R_{0}^{2}}\left\{1-\left(\frac{R_{0}}{2 \ell}\right)^{4}\right\}
$$

where $R_{0}=3 \sigma / \varepsilon$ as before. This formula will be used to check the results of the numerical study of the negative modes presented in the following section.

\section{VACUUM DECAY THROUGH O(4)-SYMMETRIC BUBBLES}

\section{A. Model and field equations}

In order to consider a wide variety of models of interest to Higgs cosmology, we generalize the gravitational action (12) 
to include nonminimal coupling between the scalar field and gravity,

$S=\int\left(-\frac{\hat{R}}{16 \pi G}+\frac{\xi}{2} \hat{R} \phi^{2}+\frac{\hat{g}^{\mu \nu}}{2}\left(\partial_{\mu} \phi\right)\left(\partial_{\nu} \phi\right)+V(\phi)\right) \sqrt{\hat{g}} d^{4} x$,

where $\xi$ is a nonminimal coupling coefficient and hats denote the choice of metric commonly referred to as the Jordan frame. We consider potentials such that $V(0)=V^{\prime}(0)=0$, $V^{\prime \prime}(0)>0$, and assume $V$ takes negative values in some interval of $\phi$ so that the bubble solutions will be noncompact. To find numerical solutions and study their perturbations, it is convenient to go to the Einstein frame by rescaling the metric:

$$
g_{\mu \nu}=\left(1-8 \pi G \xi \phi^{2}\right) \hat{g}_{\mu \nu},
$$

(for an analysis of solutions in the Jordan frame see [20,41]). The action becomes

$S=\int\left(-\frac{R}{16 \pi G}+\frac{f(\phi)^{2}}{2}\left(\partial_{\mu} \phi\right)\left(\partial^{\mu} \phi\right)+W(\phi)\right) \sqrt{g} d^{4} x$,

where

$$
f(\phi)=\frac{\sqrt{1-8 \pi G \xi(1-6 \xi) \phi^{2}}}{1-8 \pi G \xi \phi^{2}}
$$

and the modified potential is

$$
W(\phi)=\frac{V(\phi)}{\left(1-8 \pi G \xi \phi^{2}\right)^{2}} .
$$

In all the cases we will consider, $f(\phi)$ remains strictly positive. We look for $\mathrm{O}(4)$-symmetric solutions, and change slightly the form of our metric to add a lapse function:

$$
d s^{2}=N(\rho)^{2} d \rho^{2}+a(\rho)^{2} d \Omega_{I I I}^{2},
$$

The lapse function $N$ allows us to recover the full set of Einstein equations from extremization of the action, which will be convenient when deriving the eigenvalue equation. Substituting in the form of the metric (26), and integrating out over the angular variables, we obtain

$$
\begin{aligned}
S= & 2 \pi^{2} \int\left[\frac{f(\phi)^{2}}{2 N^{2}} \phi^{\prime 2}+W(\phi)\right. \\
& \left.-\frac{3}{8 \pi G}\left(\frac{1}{a^{2}}+\left(\frac{a^{\prime}}{a N}\right)^{2}\right)\right] a^{3} N d \rho,
\end{aligned}
$$

Variation with respect to $\phi$ and $N$ give the system of equations:

$$
\begin{gathered}
f(\phi)\left(f(\phi) \frac{a^{3}}{N} \phi^{\prime}\right)^{\prime}=N a^{3} W^{\prime} \\
\frac{a^{\prime 2}}{N^{2}}=1+\frac{8 \pi G}{3} a^{2}\left(\frac{f(\phi)^{2}}{2 N^{2}} \phi^{\prime 2}-W(\phi)\right) .
\end{gathered}
$$

Variation with respect to $a$ gives a Bianchi Identity. ${ }^{1}$ The system (28) and (29) can also be obtained from the full set of Einstein equations after eliminating redundancies, showing that there is no independent constraint. For boundary conditions, we look for asymptotically flat instantons, with $\phi(\infty)=\phi_{F V}$ and $a(\rho) \sim \rho$ as $\rho \rightarrow \infty$. We choose to place the centre of the instanton at $\rho=0$, where $a(0)=0$ and for regularity at the origin we must have $\phi^{\prime}(0)=0$. Equation (29) can be rewritten as:

$$
\frac{1-8 \pi G a^{2} W(\phi) / 3}{1-4 \pi G a^{2} f(\phi)^{2}\left(\partial_{a} \phi\right)^{2} / 3}=\frac{a^{\prime 2}}{N^{2}}
$$

This shows that the left-hand side, which will play an important role in the following, is always non-negative, and cannot vanish if $a$ is strictly monotonic.

The lapse function $N(\rho)$ represents some of the freedom we have to choose the coordinate gauge. We will focus on instantons where $a$ is a strictly increasing function of the distance to the center of the bubble, which allows us to choose $a$ as radial coordinate. Setting $\rho=a$, the action (27) becomes

$$
\begin{aligned}
S= & 2 \pi^{2} \int_{0}^{\infty} N a^{3}\left(\frac{f(\phi)^{2} \phi^{\prime 2}}{2 N^{2}}+W(\phi)\right) d a \\
& -\frac{3 \pi}{4 G} \int_{0}^{\infty}\left(N+\frac{1}{N}\right) a d a
\end{aligned}
$$

Variation with respect to $N$ and $\phi$ gives back the system (28) and (29), showing that no physical degree of freedom has been lost.

Since the derivative of $N$ does not appear in Eq. (31), one can express $N$ as a function of $\phi$ and $\phi^{\prime}$ :

$$
N=\left(\frac{1-4 \pi G a^{2} f(\phi)^{2} \phi^{2} / 3}{1-8 \pi G a^{2} W(\phi) / 3}\right)^{1 / 2} .
$$

This quantity is always real. The expression in the denominator is a recurring and important combination for the eigenvalue problem, hence we write

$$
Q[\phi] \equiv 1-\frac{8 \pi G}{3} a^{2} W(\phi) .
$$

Plugging Eq. (32) into Eq. (31), we obtain an unconstrained action for the scalar field $\phi$,

$$
\begin{aligned}
S= & -\frac{3 \pi}{2 G} \int_{0}^{\infty} \operatorname{sgn}(Q[\phi]) \\
& \times\left[Q[\phi]\left(1-\frac{4 \pi G a^{2}}{3} f(\phi)^{2} \phi^{\prime 2}\right)\right]^{1 / 2} a d a .
\end{aligned}
$$

\footnotetext{
${ }^{1}$ Using Eq. (28), it is equivalent to the derivative of Eq. (29).
} 

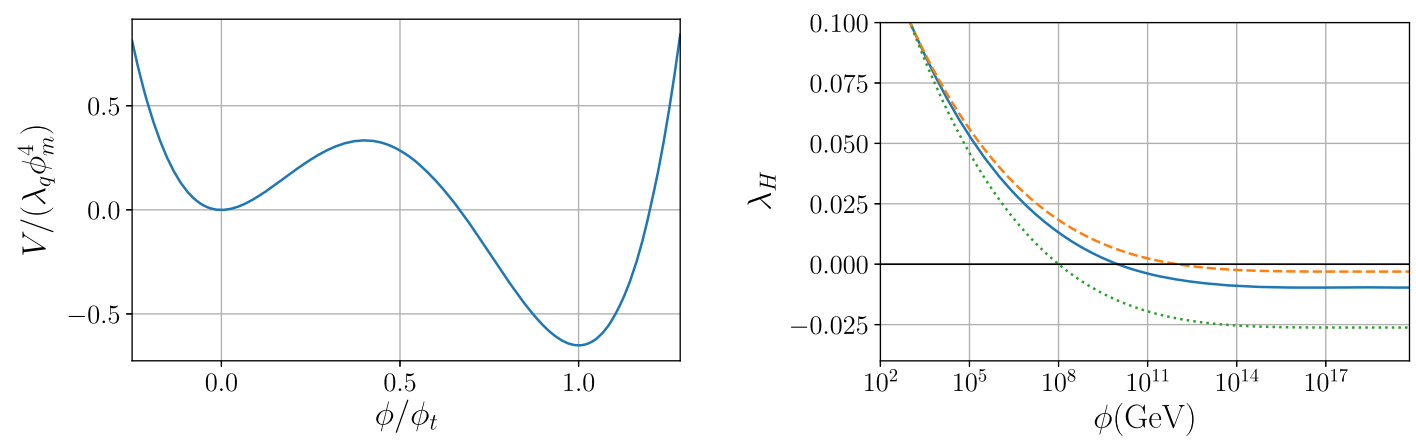

FIG. 2. Left panel: Quartic potential (38) for $\phi_{m}=M_{p} / 10, \phi_{t}=M_{p} / 4$, and $\lambda_{q}=10 / 3$. Right panel: Effective coupling for the Higgslike potential (41) for $\Lambda=10^{8} \mathrm{GeV}$ (green, dotted), $\Lambda=10^{10} \mathrm{GeV}$ (blue, continuous), and $\Lambda=10^{12} \mathrm{GeV}$ (orange, dashed), and $q$ chosen so that $\lambda\left(\phi=10^{3} \mathrm{GeV}\right)=0.1$.

Extremization of this action gives back Eq. (28) with the explicit form of $N$ given by Eq. (29).

This expression for the action can be conveniently used to derive the eigenvalue equation. To this end, let us assume we have an exact solution $\phi=\phi_{b}$. We look for a perturbed solution of the form ${ }^{2} \phi=\phi_{b}+\varphi / f\left(\phi_{b}\right)$. To quadratic order in $\varphi$, the action reads $S=S^{(0)}+S^{(2)}+O\left(\varphi^{3}\right)$, where $S^{(0)}$ is the action of the background instanton and

$$
\begin{aligned}
S^{(2)}= & 2 \pi^{2} \int_{0}^{\infty} \frac{a^{3}}{N_{b}}\left[\left(D^{2} W+\frac{8 \pi G a^{2}}{3 Q_{b}}(D W)^{2}\right.\right. \\
& \left.\left.+\frac{8 \pi G f}{3 Q_{b}} \phi_{b}{ }^{\prime} D W\right) \frac{\varphi^{2}}{2 Q_{b}}+\frac{1}{N_{b}^{2} Q_{b}} \frac{\varphi^{\prime 2}}{2}\right] d a .
\end{aligned}
$$

where $Q_{b}=Q\left[\phi_{b}\right]$, and $D=f^{-1} d / d \phi$. The simplest way to derive (35) is to regard $\phi$ as a coordinate on a one dimensional manifold with metric

$$
\mathfrak{g}=f(\phi)^{2} d \phi^{2} .
$$

The action can be evaluated in a coordinate frame with $f=1$, and then the general expression is recovered by replacing derivatives with respect to $\phi$ by the covariant derivative $D$.

The corresponding eigenvalue equation obtained from the perturbed action is

$$
\begin{aligned}
\frac{1}{N_{b} a^{3}}\left(\frac{a^{3}}{N_{b}^{3} Q_{b}} \varphi^{\prime}\right)^{\prime}= & {\left[\frac { 1 } { N _ { b } ^ { 2 } Q _ { b } } \left(D^{2} W+\frac{8 \pi G a^{2}}{3 Q_{b}}(D W)^{2}\right.\right.} \\
& \left.\left.+\frac{8 \pi G a^{2} f}{3 Q_{b}} \phi_{b}{ }^{\prime} D W\right)-\lambda\right] \varphi
\end{aligned}
$$

where $\lambda$ is the eigenvalue.

By definition, $N_{b}$ is always positive. However, $Q_{b}$ will be negative wherever $a^{2} W\left(\phi_{b}\right)>3 /(8 \pi G)$. When $Q_{b}$ is negative, the quadratic action is unbounded from below.

\footnotetext{
${ }^{2}$ Notice that $\varphi(a)$ is the geodesic distance, in the metric (36), between the perturbed and background fields.
}

(In fact, it can reach arbitrarily high negative values even for square integrable perturbations of unit $L^{2}$ norm provided the latter oscillate sufficiently fast in the region where $Q_{b}<0$.) As was shown in $[14,15]$ for instantons in de Sitter space, if the eigenmode equation has no singularity, negativity of the kinetic term implies the existence of an infinite number of negative eigenvalues.

The profusion of negative modes can be qualitatively understood as follows. In regions where the kinetic term is positive, for sufficiently large negative values of $\lambda, \varphi$ increases or decreases exponentially with $a$, with growth rate $N_{b}^{2} \sqrt{Q|\lambda|}$. If the kinetic term is positive everywhere, the boundary conditions at $a=0$ and $a \rightarrow \infty$ cannot be simultaneously satisfied. If the kinetic term reaches negative values, however, $\varphi$ becomes oscillatory in some interval, allowing us to match an exponentially decreasing function for $a \rightarrow \infty$ with a hyperbolic cosine for $a \approx 0$. More precisely, they will match provided the difference between the phases of the oscillations at both ends of the region where the kinetic term is negative exactly compensates the difference between the ratios $\varphi^{\prime} / \varphi$ for the hyperbolic cosine on the left and the exponential on the right.

It must be noted, however, that these negative modes may be physically relevant only for very thin bubbles. Indeed, negativity of the kinetic term requires that $\left|a f\left(\phi_{b}\right) \phi_{b}{ }^{\prime}\right|$ reaches values above the Planck mass. In many models, $\phi_{b}$ is limited to be less than 1 in Planck units, so that the semiclassical analysis should not break down. These negative modes may thus be physically meaningful only if $\left|a f\left(\phi_{b}\right) \phi_{b}{ }^{\prime}\right| \gg\left|\phi_{b}\right|$, i.e., either when the width of the bubble is much smaller than its radius or when $f$ is large. The latter case can occur when $\xi$ is large and negative. In the following section we will see examples that realize both of these possibilities.

\section{B. Numerical results}

We turn now to the numerical solution of the system (28) and (29) and eigenvalue equation (37) with two different shapes for the potential $V$. The first case is a quartic potential 

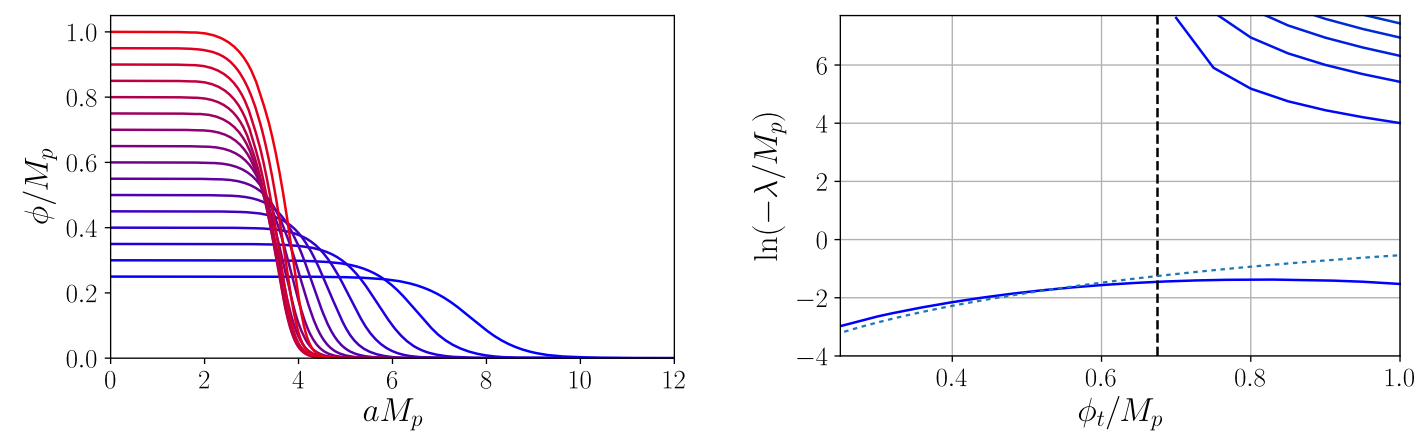

FIG. 3. Left panel: O(4)-symmetric instantons obtained with the quartic potential (38) for $\lambda_{q}=128$ and $\phi_{t} / \phi_{m}=2.5$. The value of $\phi_{t}$ in Planck units increases from blue to red. Right panel: Negative eigenvalues for these solutions. The dashed curve shows the thin wall approximation (20). The vertical dashed line shows the value $\phi_{c}$ of $\phi_{t}$ above which $Q_{b}$ takes negative values.

$V_{q}(\phi)=\frac{1}{4} \lambda_{q} \phi^{4}-\frac{1}{3} \lambda_{q}\left(\phi_{m}+\phi_{t}\right) \phi^{3}+\frac{1}{2} \lambda_{q} \phi_{m} \phi_{t} \phi^{2}$

which has been parametrized by $\phi_{m}$ and $\phi_{t}$, the field values at the maximum and the nonzero minimum respectively. The parameter $\lambda_{q}$ sets the overall scale. The origin $\phi=0$ is a false vacuum, and $\phi_{t}$ is the true vacuum when $\phi_{t}>2 \phi_{m}$. One example is shown in the left panel of Fig. 2. The numerical results do not rely on the thin-wall approximation, but the latter provides a useful the check on the results. The thin wall approximation is valid when $\phi_{t} \sim 2 \phi_{m}$.

An important derived parameter is the AdS radius of the true vacuum $\ell$. For minimal coupling $(\xi=0)$,

$$
\ell^{2}=-\frac{3 M_{p}^{2}}{V\left(\phi_{t}\right)} .
$$

For example, we expect gravitational backreaction to be important when the bubble radius is comparable to the AdS radius. In the thin-wall approximation, the flat-space bubble radius $R_{0}=3 \sigma / \epsilon$ and the ratio

$$
\frac{R_{0}}{\ell}=\frac{1}{\sqrt{2}} \frac{\phi_{t}}{M_{p}}\left(1-2 \frac{\phi_{m}}{\phi_{t}}\right)^{-1 / 2} .
$$

Note that this is independent of the overall scale parameter $\lambda_{q}$. It is possible to scan through different values of $R_{0} / \ell$ by fixing $\phi_{m} / \phi_{t}$ and scanning through different values of $\phi_{t}$.

While the quartic potential is convenient for illustrative purposes, obtaining results which may be applicable to the standard model requires a more realistic one. We thus also used a Higgs-like potential of the form

$$
\begin{aligned}
V_{H}(\phi) & =\frac{\lambda_{H}(\phi)}{4} \phi^{4}, \\
\lambda_{H}(\phi) & =q\left(\left(\ln \frac{\phi}{M_{p}}\right)^{4}-\left(\ln \frac{\Lambda}{M_{p}}\right)^{4}\right) .
\end{aligned}
$$

In this expression, $\Lambda>0$ is the scale at which the coupling and the potential vanish, and $q$ is a strictly positive number.
Like the quartic potential $V_{q}(\phi)$, this potential has a local minimum at $\phi=0$. Plots of the function $\lambda_{H}$ for three different choices of $(q, \Lambda)$ are shown in the right panel of Fig. 2. They approximate the next-to-next-to-leading order calculations reported in [23] with different values of the top quark mass.

The height of the Higgs potential barrier is small compared to $\Lambda^{4}$, making the bubble solutions shallow, with thick walls, and Higgs values inside the bubble extending beyond the barrier but do not reaching a true vacuum. The potential inside the bubble is roughly of order $\Lambda^{4}$ and the bubble size is of order $\Lambda^{-1}$, so that the "effective" value of $R_{0} / \ell$ in this case is around $\Lambda / M_{p}$.

We first work with the quadratic potential and $\xi=0$, i.e., with a minimal coupling between the scalar field $\phi$ and gravity. In Fig. 3 we show the negative eigenvalues with fixed ratio $\phi_{t} / \phi_{m}=2.5, \lambda_{q}=128$, and $\phi_{t}$ ranging from $0.25 M_{p}$ to $M_{p}$. Below a critical value $\phi_{c}$, here close to $0.67 M_{p}$, there is only one negative mode. The dashed line shows the negative mode obtained for the thin-wall approximation using (20), which agrees quite well with the numerical result despite the walls not being particularly thin.

The quantity $Q$ defined in (33) is positive for the bubble solutions with $\phi_{t}<\phi_{c}$, but for $\phi_{t}>\phi_{c}, Q$ takes negative values in a finite interval of $a$. Correspondingly, we find new negative eigenvalues, all but one going to $-\infty$ in the limit $\phi_{t} \rightarrow \phi_{c}$, in agreement with our approximate analysis in Eq. (44). The numerical evidence therefore supports the existence of infinitely many negative eigenvalues for $\phi_{t}>\phi_{c}$.

Results with nonminimal coupling are shown in Fig. 4. Here the parameters of the potential are fixed to $\phi_{m}=0.36$, $\phi_{t}=0.84$, and $\lambda_{4}=10 / 3$, and the nonminimal coupling $\xi$ is varied between -0.5 and 0.9 . At the level of the instanton solution, the main effect of a negative value of $\xi$ seems to be to increase the radius of the bubble, while a positive value increases $\phi(0)$. Its role is more dramatic when considering the negative modes: as shown in the right panel of the figure, there is a critical value $\xi_{c}$, here close to 0.2 , above 

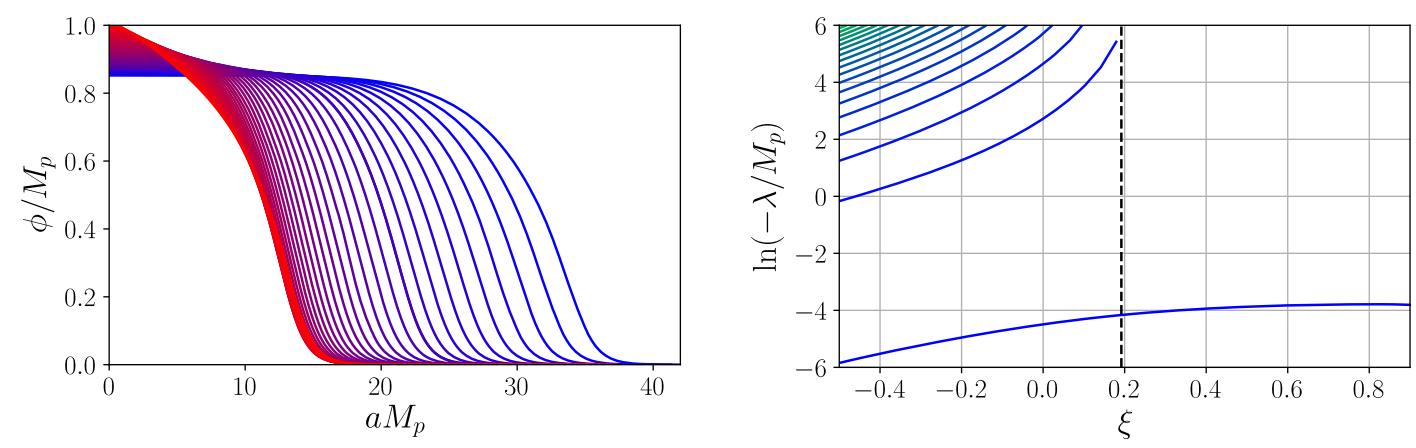

FIG. 4. Left panel: O(4)-symmetric instantons obtained with the quartic potential (38) for $\phi_{m}=0.36, \phi_{t}=0.84$, and $\lambda_{q}=10 / 3$, for different values of the nonminimal coupling $\xi$ ranging from -0.5 to 0.9 . The value of $\xi$ increases from blue to red. Right panel: Negative eigenvalues for these solutions. The vertical dashed line shows the value $\xi_{c}$ of $\xi$ below which $Q_{b}$ takes negative values.

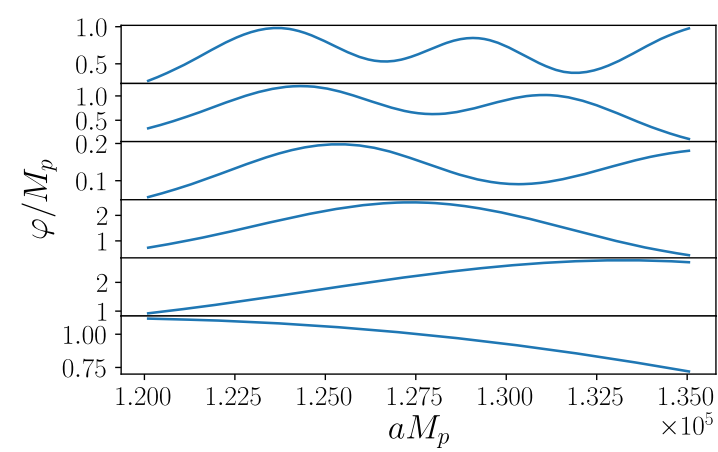

FIG. 5. Plots of the first six negative modes in the region where the kinetic term is negative. We use the Higgs-like potential (41) with $q=10^{-7}$ and $\Lambda=0.3$, and a minimal coupling $\xi=-5.3$, slightly below the critical one $\xi_{c} \approx-4.8$ for this potential. (The normalization is arbitrary.)

which only one negative mode is present, but below which there is an infinite number of them. As already noticed when varying $\phi_{t}$, the first case corresponds to a positive $Q$, while in the second case this function takes negative values in a finite interval of $a$. As in the previous case also, all but one negative eigenvalues go to negative infinity when approaching the threshold $\xi \rightarrow \xi_{c}$.

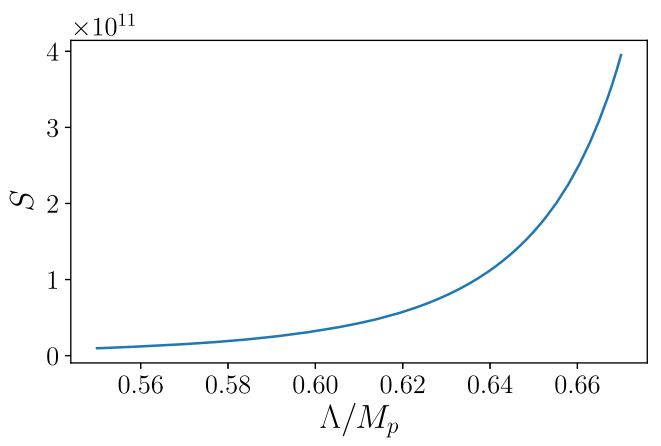

Figures 5-7 show results obtained with the potential $V_{H}$. To ease the numerical resolution, they are made with relatively high values of $\Lambda$, close to unity in Planck units. We found a similar behavior for smaller values of this parameter. In Fig. 5 are shown the first six negative modes for fixed potential and a minimal coupling $\xi$ slightly smaller than $\xi_{c}$, in the region where $Q<0$. The main information is that, as expected, negative modes are oscillatory in this region, and that the $n$th one has approximately $n / 2$ wavelengths for sufficiently large $n$.

Figures 6 and 7 shows the Euclidean action and negative eigenvalues of instantons as functions of $\Lambda$ and $\xi$ respectively, for $q=10^{-7}$. As can be seen on the left panels and more generally in Fig. 8, the Euclidean action of instantons supporting infinitely many negative modes is huge, making the transition rate negligible. We found the same holds for all parameters we tried. It thus seems that, for realistic potentials, the appearance of an infinite number of negative eigenvalues requires such a strong back-reaction from gravity on the instanton that the probability of bubble nucleation becomes negligibly small. Conversely, all instantons we found which gave non-negligible decay rates have only one negative eigenvalue.

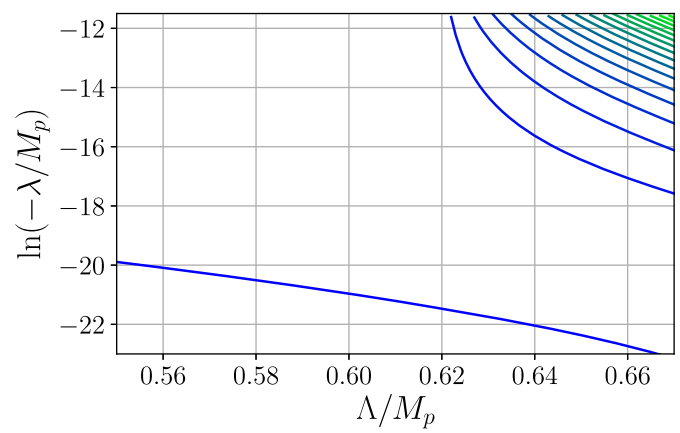

FIG. 6. Euclidean action (left panel) and negative eigenvalues (right panel) of an asymptotically flat O(4)-symmetric instanton with the Higgs potential (41) with $q=10^{-7}$ and $\xi=0$. 

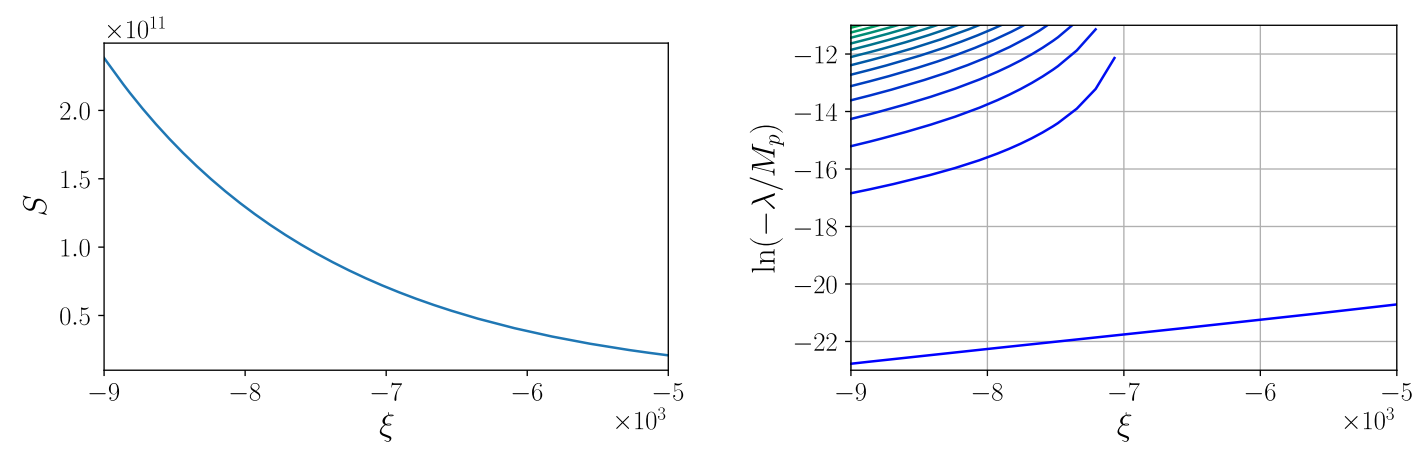

FIG. 7. Euclidean action (left panel) and negative eigenvalues (right panel) of an asymptotically flat $\mathrm{O}(4)$-symmetric instanton for the Higgs potential (41) with $q=10^{-7}$ and $\Lambda=0.5$.
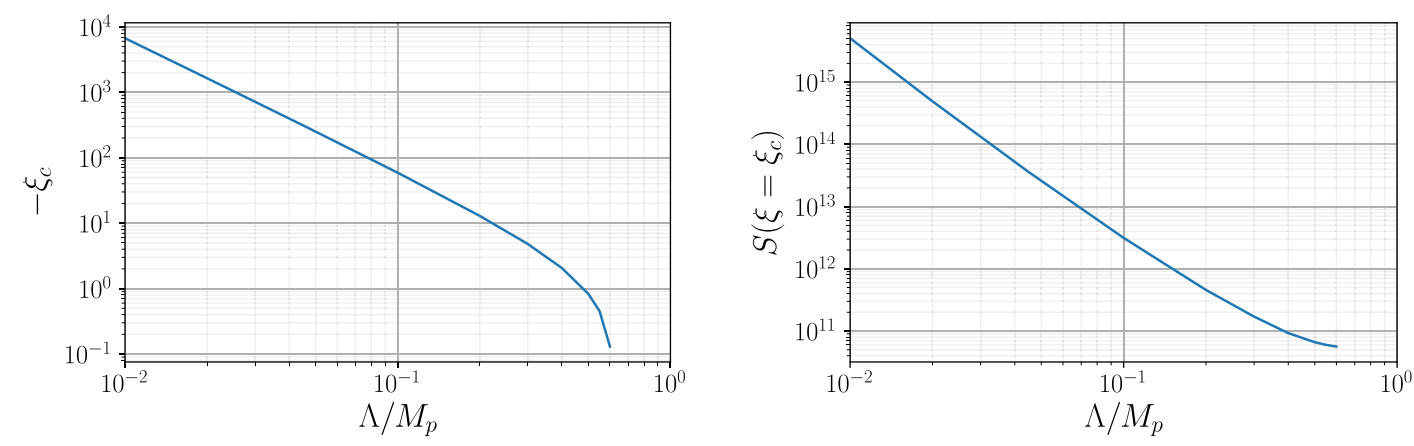

FIG. 8. Left panel: Dependence of the critical value $\xi_{c}$ of the nonminimal coupling below which an infinite number of negative modes is present in the scale $\Lambda$ at which the Higgs potential vanishes. The potential is given by (41) with $q=10^{-7}$. For larger values of $\Lambda$, $\xi_{c}$ is formally positive, but $\phi$ reaches values close to the Planck scale so that the semiclassical approximation is not expected to be valid. Right panel: Euclidean action of the critical instanton with $\xi=\xi_{c}$ for the same values of $\Lambda$.

\section{Analytical estimates}

We now mention two analytical results which help understand the numerical observations reported above. We first give an estimate of the large negative eigenvalues. For large values of $-\lambda$, one can neglect the other terms in the right-hand side of Eq. (37). Moreover, since the rate of change of $\varphi$ is proportional to $\sqrt{|\lambda|}$, we can in this limit neglect the variations of $a, Q$, and $N_{b}$. The eigenvalue equation thus becomes

$$
\varphi^{\prime \prime} \approx-Q N_{b}^{2} \lambda \varphi .
$$

Let us call $a_{-}$and $a_{+}$, the boundaries of the interval in which $Q$ is negative, ordered as $a_{-}<a_{+}$. Then, $\varphi$ is exponentially increasing or decreasing for $a>a_{+}$, and oscillating for $a_{-}<a<a_{+}$. The global solution will be decreasing at infinity provided the oscillating solution for $a$ just below $a_{+}$can be matched with the decaying one for $a>a_{+}$, with one or the opposite sign. This occurs twice each time we add one wavelength in the interval $\left[a_{-}, a_{+}\right]$. One thus expects that, for large values of $n$, the $n$th negative eigenvalue $\lambda_{n}$ satisfies

$$
\int_{a_{-}}^{a_{+}} \sqrt{Q N_{b}^{2} \lambda_{n}} d a \approx \pi n
$$

i.e.,

$$
\lambda_{n} \approx \frac{-\pi^{2} n^{2}}{\left(\int_{a_{-}}^{a_{+}} \sqrt{-Q} N_{b} d a\right)^{2}} .
$$

Notice that, since the $n$th negative mode oscillates with a wave vector proportional to $\sqrt{-\lambda_{n}}$ in the region where $B^{(0)}$ is negative, it must have an amplitude proportional to $(-\lambda)^{-1 / 4}$ to be normalized for the Klein-Gordon inner product. From the above estimate, $(-\lambda)^{-1 / 4} \propto n^{-1 / 2}$. One can thus expect that the sum of the contributions from negative modes to quadratic observables are formally divergent, which may point to an instability of the background solution or, as conjectured in [5], to a breakdown of the semiclassical approximation. However, a more detailed analysis, would be required to ascertain this. ${ }^{3}$

Next, we estimate the critical value $\xi_{c}$ below which an infinite number of negative modes are present. We assume that $-\xi \gg 1$ and that $-\xi \phi \gg 1 / \sqrt{G}$ in the relevant domain of $a$ (typically inside the bubble and including a significant fraction of the wall). In this limit, we can integrate to find the canonical field $\tilde{\phi}$, defined by

\footnotetext{
${ }^{3}$ In particular, logarithmic corrections to Eq. (44), if present, could make the series convergent.
} 


$$
\tilde{\phi}=\int d \phi f(\phi) \approx \sqrt{\frac{3}{16 \pi G}} \ln \left(1-8 \pi G \xi \phi^{2}\right) .
$$

The modified potential (25) thus becomes

$$
W(\phi) \approx e^{-8 \tilde{\phi} \sqrt{\pi G / 3}} V\left(\sqrt{\frac{1-e^{4 \tilde{\phi} \sqrt{\pi G / 3}}}{8 \pi G \xi}}\right) .
$$

Let us assume that $V$ has a zero at a value $\Lambda>0$ of $\phi$. We call $\tilde{\Lambda}$ the corresponding value of $\tilde{\phi}$. If the potential has no other typical scale, the maximum value reached by $a \tilde{\phi}^{\prime}$ should be of order $\tilde{\Lambda}$. Denoting by $\alpha$ the ratio $a \tilde{\phi}^{\prime} / \tilde{\Lambda}$, we have

$$
\min Q=1-\frac{4 \pi G}{3} \alpha^{2} \tilde{\Lambda}^{2} .
$$

Using Eq. (45), this may be rewritten as

$$
\min Q \approx 1-\frac{\alpha^{2}}{4} \ln \left(1-8 \pi G \xi \Lambda^{2}\right)^{2} .
$$

This quantity is negative provided $\xi<\xi_{c}$, where

$$
\xi_{c} \approx \frac{1-e^{2 / \alpha}}{8 \pi G \Lambda^{2}} .
$$

We thus expect $-8 \pi G \Lambda^{2} \xi_{c}$ to be of order 1 for sufficiently small values of $\Lambda^{2} G$, in accordance with results shown in the left panel of Fig. 8.

\section{VACUUM DECAY WITH A BLACK HOLE}

Now we turn to vacuum decay seeded by a microscopic black hole. The initial state consists of a region of false vacuum containing a Schwarzschild black hole. In Euclidean space, the black hole metric is periodic in the imaginary time coordinate with period $\beta=8 \pi G M$. We enforce the same boundary conditions on the bubble solution to ensure that the tunneling exponent $B=$ $S_{E}\left[\phi_{b}\right]-S_{E}\left[\phi_{\mathrm{fv}}\right]$ is finite. The black hole inside the bubble solution has a smaller mass than the original black hole.

When we take static (i.e., independent of Euclidean time $\tau)$ solutions, there is a remarkable simplification in the expression for the action which allows us to express the tunneling exponent in terms of the reduction in black hole entropy [29],

$$
B=\frac{\mathcal{A}_{S}}{4 G}-\frac{\mathcal{A}_{R}}{4 G},
$$

where $\mathcal{A}_{S}$ and $\mathcal{A}_{R}$ are the areas of the event horizon of the black hole seed and the black hole remnant. The bubble solution has a conical singularity at the horizon, but when this is properly taken into account there is no ambiguity in the action [26].

Note that in general one can find instanton solutions with a range of remnant mass for a given seed mass, but there is a unique remnant mass with lowest action. There are then two branches of solutions [26]: One branch comprises nonstatic instantons that are a variant of the CDL instanton, and continuously connected to this $O(4)$ symmetric solution in the limit $M \rightarrow 0$. The other branch occurs for seed masses larger than some critical mass, $M_{C}$, and is a "static" solution. These solutions are relevant for black holes above the Planck mass, where one can trust the semi-classical methods used. As shown in [26], the static instanton is the relevant instanton for Higgs vacuum decay, thus in this section we consider static instantons only. These have the further advantage that they are dependent only on the radial coordinate. Since the static branch is not continuously connected to the CDL instanton, we do not expect to recover the results of Sec. III in the limit $M \rightarrow 0$.

\section{A. Model and field equations}

We consider the real scalar field $\phi$ minimally coupled to gravity with the Einstein-scalar action (12). We look for spherically-symmetric bubble solutions where $\phi$ depends only on a radial coordinate $r$ and the metric has the form:

$$
d s^{2}=f(r) \mathrm{e}^{2 \delta(r)} d \tau^{2}+\frac{d r^{2}}{f(r)}+r^{2} d \Omega_{2}^{2},
$$

where $\tau$ is the Euclidean time, $f$ is a smooth positive function, and $d \Omega_{2}^{2}$ is the metric on a unit-radius, twodimensional sphere. It is also convenient to define the function $\mu$ by

$$
f(r)=1-\frac{2 G \mu(r)}{r} .
$$

The Einstein equations then give [29]

$$
\begin{gathered}
\left(r^{2} e^{\delta} f \phi^{\prime}\right)^{\prime}=r^{2} e^{\delta} V^{\prime}[\phi], \\
\mu^{\prime}=4 \pi r^{2}\left(\frac{1}{2} f \phi^{\prime 2}+V[\phi]\right), \\
\delta^{\prime}=4 \pi G r \phi^{\prime 2} .
\end{gathered}
$$

We look for asymptotically flat black hole solutions, for which $f(r)$ vanishes at the horizon $r=r_{h}$ and $\phi$ approaches the false vacuum as $r \rightarrow \infty$. Without loss of generality (up to a global rescaling of $\tau$ ), one can impose $\delta\left(r_{h}\right)=0$. The final boundary condition is given by a regularity condition at the horizon [29]:

$$
\phi^{\prime}\left(r_{h}\right)=\frac{r_{h} V^{\prime}\left[\phi\left(r_{h}\right)\right]}{1-8 \pi G r_{h}^{2} V\left[\phi\left(r_{h}\right)\right]} .
$$

In order to compute the Euclidean action, we require the Ricci scalar,

$R=-f^{\prime \prime}-3 \delta^{\prime} f^{\prime}-2 \delta^{\prime \prime} f-2 \delta^{2} f-\frac{4}{r} f^{\prime}-\frac{4}{r} \delta^{\prime} f+\frac{4 G}{r^{3}} \mu$.

Using this and performing an integration by parts, the Einstein-Hilbert action (12) becomes 


$$
\begin{aligned}
S_{\mathrm{E}}\left[\phi_{b}\right]= & 4 \pi \beta \int_{r_{h}}^{\infty} e^{\delta} r^{2}\left(-\frac{\mu^{\prime}}{4 \pi r^{2}}+\frac{f \phi^{\prime 2}}{2}+V\right) d r \\
& +\frac{\beta}{2}\left(M_{S} e^{\delta(\infty)}-M_{R}+r_{h} \mu^{\prime}\left(r_{h}\right)\right),
\end{aligned}
$$

where $\beta$ denotes the period in $\tau, M_{S} \equiv \mu(\infty)$ is the ADM mass of the initial black hole, and $M_{R} \equiv \mu\left(r_{h}\right)$ is the mass of the residual black hole. The first term vanishes when imposing Eq. (54). In Refs. [26] and [28], it was shown that including boundary terms $S_{\partial}$ gives the result

$$
S_{E}\left[\phi_{b}\right]+S_{\partial}\left[\phi_{b}\right]=-\frac{\mathcal{A}_{R}}{4 G}+\beta M_{S} .
$$

The false vacuum black hole gives

$$
S_{E}\left[\phi_{\mathrm{fvbh}}\right]+S_{\partial}\left[\phi_{\mathrm{fvbh}}\right]=-\frac{\mathcal{A}_{S}}{4 G}+\beta M_{S} .
$$

The difference produces the tunneling exponent Eq. (50).

To determine the eigenvalue equation, we write $\phi=$ $\phi_{b}+\phi_{1}, \mu=\mu_{b}+\mu_{1}$, and $\delta=\delta_{b}+\delta_{1}$, where $\left(\phi_{b}, \mu_{b}, \delta_{b}\right)$ is an exact solution of Eqs. (53)-(55). We define $f_{b} \equiv 1-2 G \mu_{b} / r$. To quadratic order, and discarding a boundary term, the action reads $S=S^{(0)}+S^{(2)}+\cdots$, where $S^{(0)}$ is the action of the instanton, dots represent higher-order terms, and

$$
\begin{aligned}
S^{(2)}= & 4 \pi \beta \int_{r_{h}}^{\infty} r^{2} \mathrm{e}^{\delta_{b}}\left[f_{b} \phi_{b}^{\prime} \phi_{1}^{\prime}-\frac{G \mu_{1}}{r} \phi_{b}^{\prime 2}+V^{\prime}\left[\phi_{b}\right] \phi_{1}-\frac{\mu_{1}^{\prime}}{4 \pi r^{2}}\right] d r \\
& +4 \pi \beta \int_{r_{h}}^{\infty} r^{2} \mathrm{e}^{\delta_{b}} \delta_{1}\left[f_{b} \phi_{b}^{\prime} \phi_{1}^{\prime}-\frac{G \mu_{1}}{r} \phi_{b}^{\prime 2}+V^{\prime}\left[\phi_{b}\right] \phi_{1}-\frac{\mu_{1}^{\prime}}{4 \pi r^{2}}\right] d r \\
& +4 \pi \beta \int_{r_{h}}^{\infty} r^{2} \mathrm{e}^{\delta_{b}}\left[\frac{f_{b}}{2} \phi_{1}^{\prime 2}-\frac{2 G \mu_{1}}{r} \phi_{b}^{\prime} \phi_{1}^{\prime}+V^{\prime \prime}\left[\phi_{b}\right] \frac{\phi_{1}^{2}}{2}\right] d r .
\end{aligned}
$$

The first integral vanishes with the boundary condition $\mu_{1}(\infty)=0$. Variation of $S^{(2)}$ with respect to $\mu_{1}$ gives the constraint

$$
\delta_{1}^{\prime}=8 \pi G r \phi_{b}^{\prime} \phi_{1}^{\prime}
$$

Variation with respect to $\delta_{1}$ gives

$$
\mu_{1}^{\prime}=4 \pi r^{2}\left[f_{b} \phi_{b}^{\prime} \phi_{1}^{\prime}+V^{\prime}\left[\phi_{b}\right] \phi_{1}-\frac{G \mu_{1}}{r} \phi_{b}^{\prime 2}\right] .
$$

Using equations (53)-(55) and assuming the boundary condition $\mu_{1}(\infty)=\phi_{1}(\infty)=0$, this becomes

$$
\mu_{1}=4 \pi r^{2} f_{b} \phi_{b}{ }^{\prime} \phi_{1}
$$

Using Eqs. (62) and (64), the quadratic action becomes

$$
\begin{aligned}
S^{(2)}= & 4 \pi \beta \int_{r_{h}}^{\infty} r^{2} e^{\delta_{b}}\left[\frac{f_{b}}{2} \phi_{1}^{\prime 2}-8 \pi G r f_{b} \phi_{b}^{\prime 2} \phi_{1} \phi_{1}{ }^{\prime}\right. \\
& \left.+V^{\prime \prime}\left[\phi_{b}\right] \frac{\phi_{1}^{2}}{2}\right] d r .
\end{aligned}
$$

Integrating by parts the second term inside the square brackets and using again Eqs. (53)-(55), one obtains

$$
S^{(2)}=4 \pi \beta \int_{r_{h}}^{\infty} r^{2} e^{\delta_{b}}\left[\frac{f_{b}}{2} \phi_{1}^{\prime 2}+\mathcal{V}(r) \frac{\phi_{1}^{2}}{2}\right] d r
$$

where

$$
\begin{aligned}
\mathcal{V}(r) \equiv & V^{\prime \prime}\left[\phi_{b}\right]+16 \pi G r V^{\prime}\left(\phi_{b}\right) \phi_{b}{ }^{\prime} \\
& -8 \pi G r\left(f_{b}^{\prime}+\delta_{b}^{\prime} f_{b}+\frac{f_{b}}{r}\right) \phi_{b}{ }^{\prime 2}
\end{aligned}
$$

The eigenvalue equation from the action (66) is

$$
\frac{e^{-\delta_{b}}}{r^{2}} \frac{d}{d r}\left(r^{2} e^{\delta_{b}} f_{b} \phi_{1}{ }^{\prime}\right)=(\mathcal{V}(r)-\lambda) \phi_{1}
$$

Contrary to the O(4)-symmetric case studied in Sec. III, here the prefactor of the kinetic term in the quadratic action, $r^{2} e^{\delta_{b}} f_{b}$, is always positive outside the horizon. Since the presence of an infinite number of negative modes in the previous case was due to the kinetic term reaching negative values, we conjecture they do not arise in the present model. ${ }^{4}$ This conjecture is supported by the numerical investigation of Eq. (68) discussed below.

\section{B. Numerical results}

We solved the system (53)-(55) and the eigenvalue equation (68) in the two potentials (38) and (41). Results for the tunneling exponent $B$ and negative eigenvalues $\lambda$ are shown in Figs. 9 and 10.

\footnotetext{
${ }^{4}$ As explained in Sec. III, a nonminimal coupling to gravity is equivalent to a change of potential. In the present case, this will not change the sign of the kinetic term.
} 

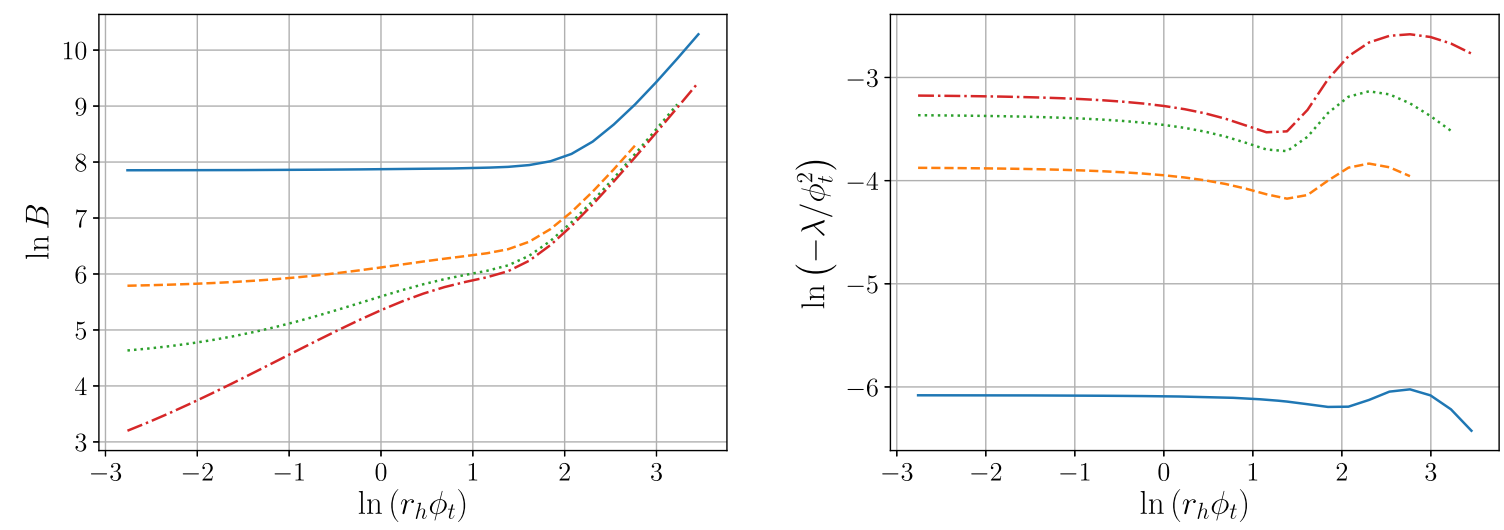

FIG. 9. Tunneling exponent (50) for seeded nucleation (left panel) and negative eigenvalue (right panel) of the instanton with black hole for the quartic potential (38) with the parameters $a_{4}=1, \phi_{t}=2 \alpha$ and $\phi_{m}=0.6 \alpha$, where $\alpha=1$ (blue), $10^{-1 / 4}$ (orange), $10^{-1 / 2}$ (green), and $10^{-1}$ (red).
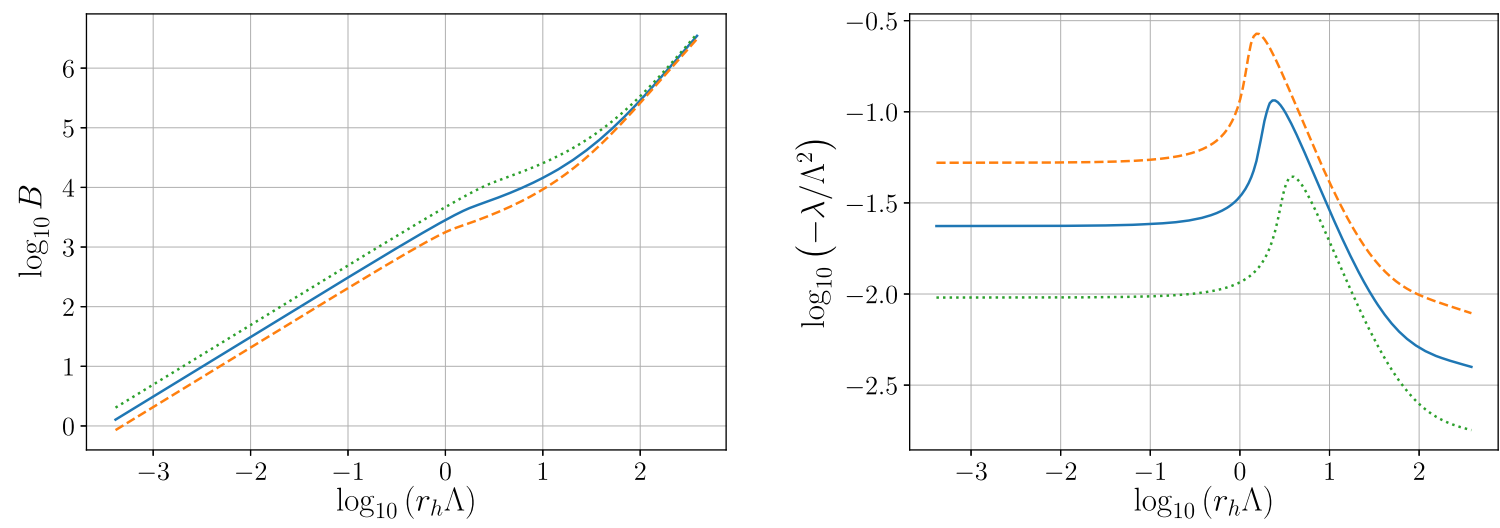

FIG. 10. Tunneling exponent (50) for seeded nucleation (left panel) and negative eigenvalue (right panel) obtained for the Higgs potential (41) for the same values of the parameters as in Fig. 2, right panel.

Notice that in the case of the quartic potential (38) we have an approximate symmetry when the effects of gravity are sufficiently small. Indeed, neglecting the term in $\mu^{\prime}$, Eq. (58) is invariant under $\phi \rightarrow \eta \phi, \phi_{m} \rightarrow \eta \phi_{m}, \phi_{t} \rightarrow \eta \phi_{t}$, $r \rightarrow r / \eta, \beta \rightarrow \beta / \eta, \mu \rightarrow \eta \mu$ at fixed $a_{4}$ for any $\eta>0$. The differences between the curves shown in each panel of Fig. 9 are thus entirely due to the gravitational backreaction, which has the tendency to increase the tunneling exponent $B$ and decrease the absolute value of $\lambda$.

For both potentials, in the whole range of parameters we tried we always found only one negative mode, as could be expected from the facts that the kinetic term in the eigenvalue equation (68) is positive definite outside the horizon and the background solution has no node. This is the main result of our work, and suggests that the static instantons with black holes found in $[26,28,29]$ can be safely interpreted as the dominant contribution to the decay rate of the false vacuum in the presence of small black holes.

To confirm and better understand this result, it is useful to define the coordinate $x$ by $d x=r^{2} e^{\delta_{b}} d r$. The eigenvalue equation (68) then becomes

$$
\frac{d}{d x}\left(r^{4} e^{2 \delta_{b}} f_{b} \frac{d \phi_{1}}{d x}\right)=(\mathcal{V}-\lambda) \phi_{1}
$$

This has the form of a Schrödinger equation, for which nodal theorems apply. In particular, the results of [44] (see also [45]) motivate that the number of negative eigenvalues is equal to the number of nodes of the solution with $\lambda=0$ satisfying the correct boundary condition at the horizon. ${ }^{5}$ Three solutions corresponding to different values of $r_{h}$ are shown in Fig. 11 for the Higgs-like potential with $\Lambda=10^{-10}$. Each of them has only one node, which confirms there should exist one and only one negative mode over each instanton.

\footnotetext{
${ }^{5}$ This does not constitute a rigorous proof, however, for two reasons. First, the function $f_{b}$ vanishes at $r=r_{h}$, while the theorem proved in [44] applies to uniformly positive functions. Second, the boundary condition at the origin used in this reference is $\phi_{1}(0)=0$ instead of $\phi_{1}{ }^{\prime}\left(r_{h}\right)=0$. We expect that these two differences do not change the result, but have so far not been able to prove it rigorously.
} 


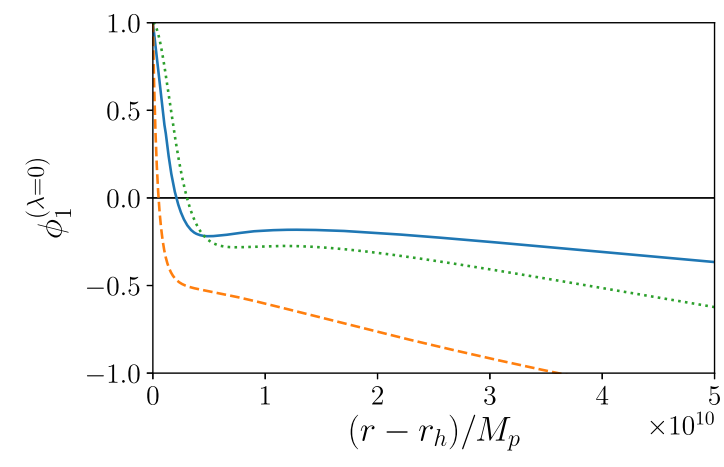

FIG. 11. Solutions of Eq. (50) with $\lambda=0$ for the Higgs-like potential (41) with $\Lambda=10^{-10}$. The Schwarzschild radius $r_{h}$ is equal to $0.1 \Lambda^{-1}$ (orange), $\Lambda^{-1}$ (blue), and $10 \Lambda^{-1}$ (green).

\section{CONCLUSIONS}

We have studied negative modes of instantons in two different setups: First the case of asymptotically flat $\mathrm{O}(4)$-symmetric "Coleman-de Luccia" type instantons, including a nonminimal coupling of the scalar, and second the case of black hole catalysed vacuum decay developed in [27-29].

For the $\mathrm{O}(4)$-symmetric asymptotically flat instantons, we explored a wide range of parameter space with a conventional quartic potential, as well more phenomenologically realistic analytic fit to the standard model Higgs potential. For any value of the nonmimimal coupling parameter $\xi$, it is always possible to find a region of parameter space in the potential that has an infinite tower of negative modes for the corresponding instanton, however, these parameter values correspond to energies close to the Planck scale.

For the black hole instantons, the kinetic term of the quadratic action is always positive outside the horizon, and we confirmed numerically that there is always only one negative mode. Although we did not explicitly consider a nonminimal coupling here, this would amount to a change of potential which does not affect the sign of the kinetic term. We thus expect the number of negative modes to be still equal to 1 when including it.

As already noted in $[14,15,46]$, the infinite tower of negative modes arising when the kinetic term of the quadratic action reaches negative values remains mysterious, although it is intriguing that the tower of modes appear approximately at the self-compactification scale corresponding to a domain wall topological defect of tension $\sigma[47,48]$. In Sec. III C, using an analytical estimate for the large negative eigenvalues in the $\mathrm{O}(4)$-symmetric case, we argue that these infinite negative modes induce a divergence in quadratic observables, which seems to support the argument that they may signal a breakdown of the semiclassical approximation.

A more precise answer may require studying timedependent perturbations to see if these additional modes manifest themselves, maybe as an instability of the instanton. However, assuming asymptotic flatness, both in the $\mathrm{O}(4)$-symmetric and black-hole case, we found that realistic instantons always have exactly one negative mode. It thus seems safe to interpret the lowest-action instanton as giving the leading contribution to the tunneling rate.

\section{ACKNOWLEDGMENTS}

This research was supported in part by the Leverhulme Trust, in part by Perimeter Institute for Theoretical Physics, and in part by STFC. K.M., F.M., and I. G. M. would also like to thank Perimeter Institute for hospitality while this work was in progress. R. G., F. M., and I. G. M. are supported by Leverhulme Grant No. RPG-2016-233, R. G. is also supported by Perimeter Institute, and K. M. is supported by an STFC studentship. Research at Perimeter Institute is supported by the Government of Canada through the Department of Innovation, Science and Economic Development and by the Province of Ontario through the Ministry of Research and Innovation.
[1] S. R. Coleman, The fate of the false vacuum. 1. Semiclassical theory, Phys. Rev. D 15, 2929 (1977); Erratum, Phys. Rev. D 16, 1248(E) (1977).

[2] C. G. Callan and S. R. Coleman, The fate of the false vacuum. 2. First quantum corrections, Phys. Rev. D 16, 1762 (1977).

[3] S. R. Coleman, Quantum tunneling and negative eigenvalues, Nucl. Phys. B298, 178 (1988).

[4] S. R. Coleman and F. De Luccia, Gravitational effects on and of vacuum decay, Phys. Rev. D 21, 3305 (1980).
[5] G. V. Lavrelashvili, V. A. Rubakov, and P. G. Tinyakov, Tunneling transitions with gravitation: Breaking of the quasiclassical approximation, Phys. Lett. 161B, 280 (1985).

[6] T. Tanaka and M. Sasaki, False vacuum decay with gravity: Negative mode problem, Prog. Theor. Phys. 88, 503 (1992).

[7] J. Garriga, Nucleation rates in flat and curved space, Phys. Rev. D 49, 6327 (1994).

[8] S. Gratton and N. Turok, Cosmological perturbations from the no boundary Euclidean path integral, Phys. Rev. D 60, 123507 (1999). 
[9] G. V. Lavrelashvili, Negative mode problem in false vacuum decay with gravity, Nucl. Phys. B, Proc. Suppl. 88, 75 (2000).

[10] T. Tanaka, The no-negative mode theorem in false vacuum decay with gravity, Nucl. Phys. B556, 373 (1999).

[11] S. Gratton and N. Turok, Homogeneous modes of cosmological instantons, Phys. Rev. D 63, 123514 (2001).

[12] A. Khvedelidze, G. V. Lavrelashvili, and T. Tanaka, Cosmological perturbations in a Friedmann-RobertsonWalker model with a scalar field and false vacuum decay, Phys. Rev. D 62, 083501 (2000).

[13] G. V. Dunne and Q.-h. Wang, Fluctuations about cosmological instantons, Phys. Rev. D 74, 024018 (2006).

[14] H. Lee and E. J. Weinberg, Negative modes of Coleman-De Luccia bounces, Phys. Rev. D 90, 124002 (2014).

[15] M. Koehn, G. Lavrelashvili, and J.-L. Lehners, Towards a solution of the negative mode problem in quantum tunnelling with gravity, Phys. Rev. D 92, 023506 (2015).

[16] I. V. Krive and A. D. Linde, On the vacuum stability problem in gauge theories, Nucl. Phys. 117, 265 (1976).

[17] N. Cabibbo, L. Maiani, G. Parisi, and R. Petronzio, Bounds on the fermions and Higgs boson masses in grand unified theories, Nucl. Phys. B158, 295 (1979).

[18] H. David Politzer and S. Wolfram, Bounds on particle masses in the Weinberg-Salam model, Phys. Lett. 82B, 242 (1979).

[19] G. Isidori, G. Ridolfi, and A. Strumia, On the metastability of the standard model vacuum, Nucl. Phys. B609, 387 (2001).

[20] A. Rajantie and S. Stopyra, Standard model vacuum decay with gravity, Phys. Rev. D 95, 025008 (2017).

[21] A. Andreassen, W. Frost, and M. D. Schwartz, Scale invariant instantons and the complete lifetime of the standard model, Phys. Rev. D 97, 056006 (2018).

[22] V. Branchina, F. Contino, and A. Pilaftsis, Protecting the stability of the EW vacuum from planck-scale gravitational effects, Phys. Rev. D 98, 075001 (2018).

[23] G. Degrassi, S. Di Vita, J. Elias-Miro, J. R. Espinosa, G. F. Giudice, G. Isidori, and A. Strumia, Higgs mass and vacuum stability in the standard model at NNLO, J. High Energy Phys. 08 (2012) 098.

[24] D. Buttazzo, G. Degrassi, P. Paolo Giardino, G. F. Giudice, F. Sala, A. Salvio, and A. Strumia, Investigating the nearcriticality of the Higgs boson, J. High Energy Phys. 12 (2013) 089.

[25] K. Blum, R. Tito D'Agnolo, and J. Fan, Vacuum stability bounds on Higgs coupling deviations in the absence of new bosons, J. High Energy Phys. 03 (2015) 166.

[26] R. Gregory, I. Moss, and B. Withers, Black holes as bubble nucleation sites, J. High Energy Phys. 03 (2014) 081.

[27] P. Burda, R. Gregory and I. Moss, Gravity and the Stability of the Higgs Vacuum, Phys. Rev. Lett. 115, 071303 (2015).
[28] P. Burda, R. Gregory, and I. Moss, Vacuum metastability with black holes, J. High Energy Phys. 08 (2015) 114.

[29] P. Burda, R. Gregory, and I. Moss, The fate of the Higgs vacuum, J. High Energy Phys. 06 (2016) 025.

[30] R. Gregory and I. G. Moss, The fate of the Higgs vacuum, Proc. Sci. ICHEP2016 (2016) 344.

[31] N. Tetradis, Black holes and Higgs stability, J. Cosmol. Astropart. Phys. 09 (2016) 036.

[32] P. Chen, G. Domènech, M. Sasaki, and D.-h. Yeom, Thermal activation of thin-shells in anti-de Sitter black hole spacetime, J. High Energy Phys. 07 (2017) 134.

[33] K. Mukaida and M. Yamada, False vacuum decay catalyzed by black holes, Phys. Rev. D 96, 103514 (2017).

[34] D. Gorbunov, D. Levkov, and A. Panin, Fatal youth of the Universe: Black hole threat for the electroweak vacuum during preheating, J. Cosmol. Astropart. Phys. 10 (2017) 016.

[35] P. Chen, M. Sasaki,, and D.-h. Yeom, Hawking radiation as instantons, arXiv:1806.03766.

[36] A. Aguirre and M.C. Johnson, Two tunnels to inflation, Phys. Rev. D 73, 123529 (2006).

[37] A. Aguirre, T. Banks, and M. Johnson, Regulating eternal inflation. II. The great divide, J. High Energy Phys. 08 (2006) 065.

[38] R. Bousso, B. Freivogel, and M. Lippert, Probabilities in the landscape: The decay of nearly flat space, Phys. Rev. D 74, 046008 (2006).

[39] G. Isidori, V. S. Rychkov, A. Strumia, and N. Tetradis, Gravitational corrections to standard model vacuum decay, Phys. Rev. D 77, 025034 (2008).

[40] A. Salvio, A. Strumia, N. Tetradis, and A. Urbano, On gravitational and thermal corrections to vacuum decay, J. High Energy Phys. 09 (2016) 054.

[41] A. Rajantie and S. Stopyra, Standard model vacuum decay in a de Sitter background, Phys. Rev. D 97, 025012 (2018).

[42] S. R. Coleman, V. Glaser, and A. Martin, Action minima among solutions to a class of Euclidean scalar field equations, Commun. Math. Phys. 58, 211 (1978).

[43] D. Garfinkle and R. Gregory, Corrections to the thin wall approximation in general relativity, Phys. Rev. D 41, 1889 (1990).

[44] H. Amann and P. Quittner, A nodal theorem for coupled systems of Schrödinger equations and the number of bound states, J. Math. Phys. (N.Y.) 36, 4553 (1995).

[45] P. Hartman, Ordinary Differential Equations, 2nd ed. (Society for Industrial and Applied Mathematics, Philadelphia, 2002).

[46] L. Battarra, G. Lavrelashvili, and J.-L. Lehners, Negative modes of oscillating instantons, Phys. Rev. D 86, 124001 (2012).

[47] G. W. Gibbons, Global structure of supergravity domain wall space-times, Nucl. Phys. B394, 3 (1993).

[48] F. Bonjour, C. Charmousis, and R. Gregory, Thick domain wall universes, Classical Quantum Gravity 16, 2427 (1999). 